\title{
Software of Cyclic Analysis and Forecasting in the Example of Stock Prices
}

\author{
Vladimir D. Bogatyryov, Anastasiya Yu. Sitnikova \\ Department of Economics, Samara State Aerospace University, Samara, Russian Federation.
}

Keywords: Economic cycle, wave, technical analysis, trade indicator.

Abstract. The article deals with the cyclicity of economic processes and phenomena, the development of cyclic analysis algorithm and its implementation as software.

\section{Introduction}

In the course of time in business systems both qualitative features reflecting relationships between people during production, distribution and exchange of material welfares and quantitative features such as amount of products, employment rate, producing capacity utilization, price level, profit, interest rate, money supply, velocity of money change. As these changes accumulate, forces that gave them a new stimulus for development increase, and business system starts to move in opposite direction. So development of business processes has a cyclic nature: growth is accompanied by recession without fail, and after that revival and new growth.

The reasons of business-cycles may be both internal (fluctuation of aggregate expenditures, renewal of basic production assets, consumption and investments, government economic policy) and external factors (wars, revolutions, first-rate discoveries and inventions, demographic processes). Influence of these factors on business system depends on its internal structure and capacity of adaptation to changes. So business cycle is a result of internal self-development processes interaction inside of business system and external impulses outside of it.

\section{Knowledge of Problem}

The problem of cyclicity has always attracted economists and it's one of the most disputable and little-studied problem. There are more then 1300 types of cyclicity depending on its duration (periodicity) and motive forces.

Economists had researched crisis phenomenon and cyclicity problem in economics from the beginning of 19th century. Many famous scientists studied economic cycles: J. Sismondi [1], K. Marx [2], C. Juglar, J. Kitchin [3], S. Kuznets, J. Schumpeter [4], A. Gelfand (Parvus), J. van Gelderen, S. de Volff, N. Kondratiev, R. N. Elliott [5].

There are some theories of cyclicity which are in force today - political business cycle theory, equilibrium business cycle theory, real business cycle theory, impulse-spread business cycle theory. Each of them in a varying degree of reliability reflects the reasons of cyclic fluctuations. But there is not common theory which was approved by all schools of economic thought. Economists don't have common opinion about economic cycle duration and isolation methods of economic cycles from economic factor movement.

Long-, medium- and short-term cycles interact supplemented each other, and compile common process of economic development, and its dynamic has complex form. There is not theoretically substantiated method of different-term cycles isolation.

J. Murphy in his book "Technical Analysis of the Finantial Markets" suggested using cyclic analysis in combination with other methods of technical analysis to determine the moments of market trends' beginnings and endings [6].

The Principle of Summation holds that all price movement is the simple addition of all active cycle with different lengths. Thus, each of those individual cycles could be isolated and measured, and then by continuing all cycles into the future and summing them back together again, we could find the future price dynamics and the moment of future price maximum or minimum [6]. 
But it's rather difficult to isolate each dominant cycle. There are numerical methods, which represent the price in a convenient form for harmonic analysis. These methods involve complex mathematical calculations and are not used by simple traders in practice because of unavailability of software products using these methods. Therefore, most traders use the "visual inspection" method.

Thus, the cycle isolation of the difficult state of the financial markets is an important and actual problem, so there are no scientifically validated methods of security quotations cycle isolation.

\section{Cyclic Analysis Algorithm}

Next, algorithm of embedding of cyclic analysis is proposed, it allows to isolate of cyclical fluctuations using above-stated approach. As stated above any dynamic series (eg., changes in the stock price) can be represented as the sum of long-term trend, harmonics (Fig. 1) and random component:

$$
\tilde{Y}=Y^{t r}+\sum_{k=1}^{K} Y_{k}^{\text {garm }}+\varepsilon
$$

where $Y^{t r}-$ linear trend or long-term component, which describes the basic tendency; $Y_{k}^{\text {garm }}$ harmonic; $\varepsilon$-random component.

For isolation of cycles from dynamic series $Y=\left(Y_{0}, \ldots, Y_{t}, \ldots, Y_{N-1}\right)$, consisting of $\mathrm{N}$ data points made over an equal time interval (hour, day, month, year, etc.) $-{ }_{t \in[0 ; N-1]}$, we could use cyclic analysis. According to it the harmonic is presented as:

$$
\begin{gathered}
Y_{k}^{\text {garm }}=\left(Y_{k, 0}^{\text {garm }}, \ldots, Y_{k, t}^{\text {garm }}, \ldots, Y_{k, N-1}^{\text {garm }}\right) \\
Y_{k, t}^{\text {garm }}=A_{k} \sin \left(\omega_{k} t+\varphi_{0 k}\right),
\end{gathered}
$$

where $A_{k}$ - movement amplitude determined by the formula:

$$
A_{k}=\frac{\sum_{t=0}^{N-1} Y_{t} \sin \left(\omega_{k} t+\varphi_{0 k}\right)}{\sum_{t=0}^{N-1} \sin ^{2}\left(\omega_{k} t+\varphi_{0 k}\right)} ;
$$

$\omega_{k}$ - movement frequency $\omega_{k}=\frac{2 \pi}{T_{k}} ; T_{k}$ - movement period of harmonic; $\varphi_{0 k}$ - initial movement phase, i.e. movement phase in initial time point $t=0$, which is determined by the formula: $\varphi_{0 k}=\frac{\left(T_{k}-2 \tau_{k}\right) \pi}{T_{k}}$; in angular units; $\tau_{k}$ - initial movement phase in time units, it is included in interval $\left[0 ; T_{k}\right)$.

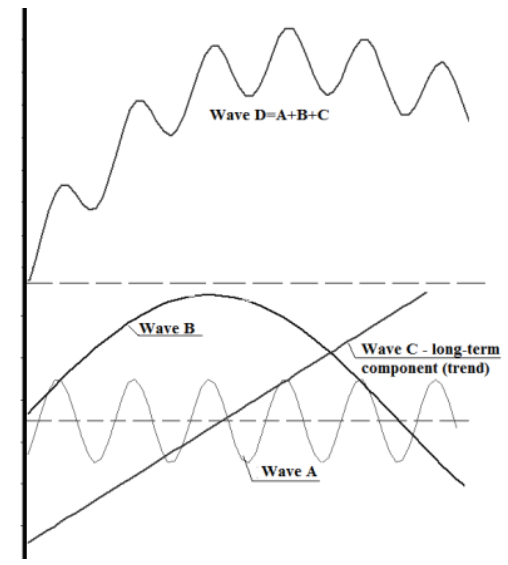

Fig. 1. Dynamic series as a sum of some waves with different periods.

The algorithm stages are presented in Fig. 2. 
At the first stage there is choosing of initial dynamic series $Y=\left(Y_{0}, \ldots, Y_{t}, \ldots, Y_{N-1}\right)$, consisting of $\mathrm{N}$ data points, for cycle isolation.

The second stage is determination of primary model for initial data exposition. For this purpose it is necessary to isolate a trend. According to the method of ordinary least squares (OLS) coefficients $a$ and $b$ of linear regression $y_{i}=a+b x_{i}$.

So, on this stage primary model describing dynamic series $Y=\left(Y_{0}, \ldots, Y_{t}, \ldots, Y_{N-1}\right)$ is a linear trend: $\tilde{Y}=Y^{t r}$.

The third stage is de-trending of initial dynamic series. For tendency remove received trend ${ }^{Y^{t r}}$ is subtracted from initial series $Y=\left(Y_{0}, \ldots, Y_{t}, \ldots, Y_{N-1}\right)$. Dynamic series centered on the $\mathrm{X}$-axis has the form: $\stackrel{o}{Y}=Y-Y^{t r}$.

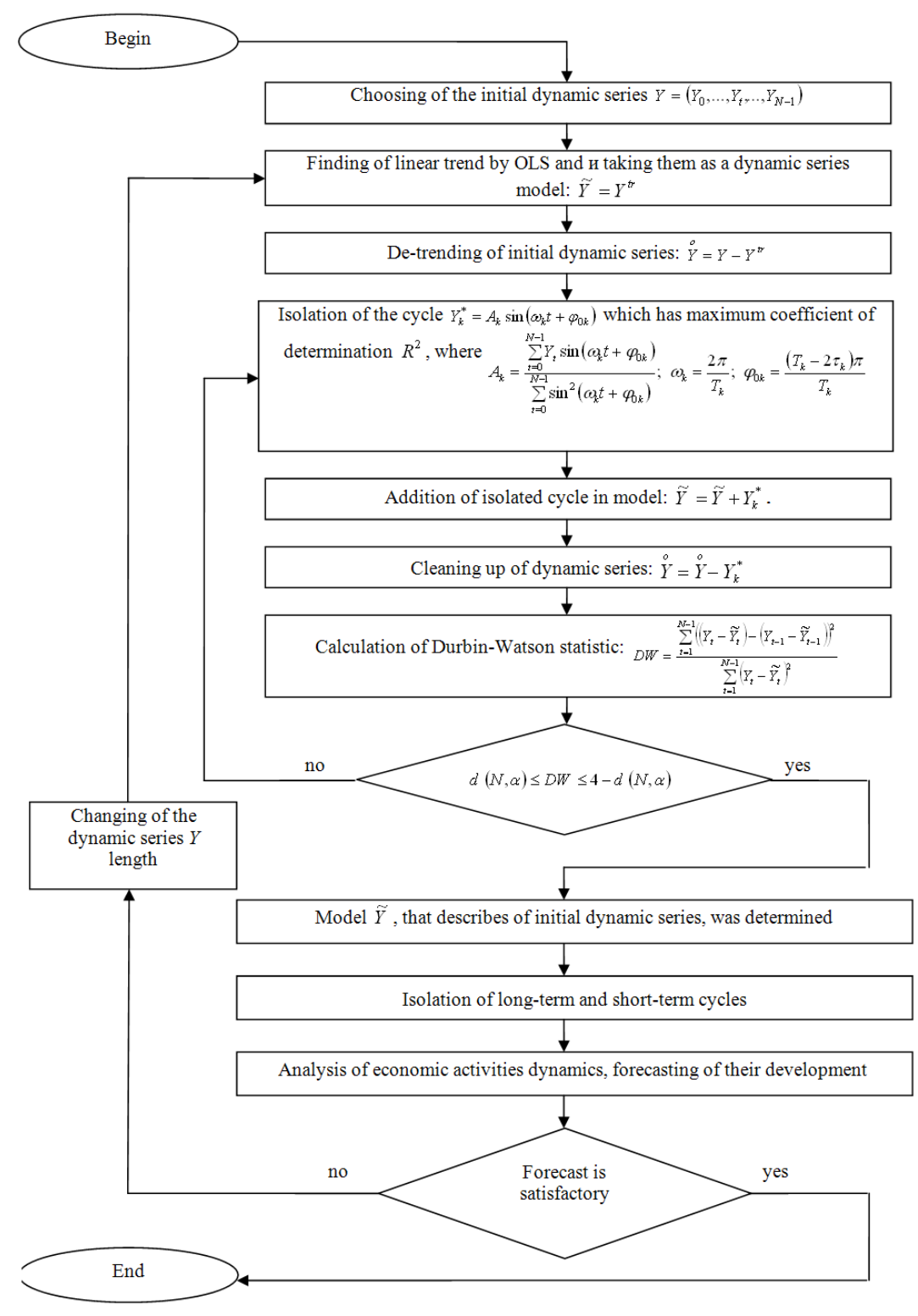

Fig. 2. Algorithm of cyclic analysis.

At the fourth stage in dynamic series $\stackrel{o}{Y}$ harmonic $Y_{k}^{\text {gamm }}$ with parameters $T_{k} \in[2 ; N]$ and $\tau_{k} \in\left[0 ; \frac{T_{k}}{2}\right]$. The harmonic provides the maximum coefficient of determination $R^{2}$.

So for harmonic $Y_{k}^{\text {garm }}$ coefficient of determination $R^{2}$ is calculated by formula: 


$$
R^{2}=1-\frac{\sum_{t=0}^{N-1}\left(Y_{t}-Y_{t k}^{\text {garm }}\right)^{2}}{\sum_{t=0}^{N-1}\left(Y_{t}-\bar{Y}\right)^{2}} .
$$

At the fifth stage the harmonic $Y_{k}^{*}=Y_{k}^{g a m}$ isolated in each cycle with the maximum $R^{2}$ is added to model of dynamic series $Y=\left(Y_{0}, Y_{1}, \ldots, Y_{N-1}\right)$ :

$$
\tilde{Y}=\tilde{Y}+Y_{k}^{*}
$$

At the sixth stage the harmonic ${ }^{Y_{k}^{*}}$ is subtracted from centered series $\stackrel{\circ}{Y}$. There is new «cleared» series $\stackrel{o}{Y}=\stackrel{o}{Y}-Y_{k}^{*}$.

The seventh stage is calculation of Durbin-Watson statistic [7] on the basis of $\operatorname{series}^{\stackrel{o}{Y}}$, which was found on the stage 6 :

$$
D W=\frac{\sum_{t=1}^{N-1}\left(\left(Y_{t}-\tilde{Y}_{t}\right)-\left(Y_{t-1}-\tilde{Y}_{t-1}\right)\right)^{2}}{\sum_{t=1}^{N-1}\left(Y_{t}-\tilde{Y}_{t}\right)^{2}}
$$

At the eighth the calculated DW value is compared with tabular value. As a result it is possible to make up a conclusion about presence (absence) of autocorrelation in residuals of first order with the some level of statistical significance. If the condition is met:

$$
d(N, \alpha) \leq D W \leq 4-d(N, \alpha),
$$

Algorithm ends. In the above condition $d(N, \alpha)$ - tabular value of Durbin-Watson statistic (for the level of statistical significance $\alpha$, series length $\mathrm{N}$ and one explanatory variable).

If the condition is not met there are association in recently "cleared" series $\stackrel{o}{Y}$. For finding of the associations harmonic isolation procedure is realized again (stage 4-stage 8).

If the condition is met then residuals of "cleared" series $\stackrel{\circ}{Y}$ have random nature, i.e. all associations of series $Y=\left(Y_{0}, \ldots, Y_{t}, \ldots, Y_{N-1}\right)$ were isolated, and final model of dynamic series is $\tilde{Y}$.

Knowing the dependence $\tilde{Y}$ on the time interval ${ }^{t \in[0 ; N-1]}$, we can calculate the values $\tilde{Y}$ at the next time interval $t \in[N ; N+m]$, were $\mathrm{m}$ - forecast horizon. The larger forecast horizon, the lower the accuracy of the prediction.

\section{Software of Cyclic Analysis}

The proposed algorithm was realized as software designed in Delphi language. The software allows to carry out technical analysis, to optimize the parameters of the standard trading indicators, to determine the signals of buying / selling securities and to calculate a profitability of using of a trading indicator. Moreover, the program allows to realize of cyclic analysis of quotations securities with detection of harmonics.

Data in the form of a *prn file format that contains information about the prices of opening and closing, highest and lowest prices of trade sessions and trade volume of securities of a particular company received by a user on the Internet can be represented graphically by means of the developed software.

The most common and useful way of graphical representation of the changes in stock prices today is the "Japanese candlestick" (Fig. 3).

The software realizes the algorithm of cyclic analysis which was described above. On the fig.4 the example of realizing of cyclic analysis of "Gazprom" stock prices, which are imaged in the top 
part of panel. In the lower part of figure there are isolated cycles, their movement period (T) and amplitude (A).

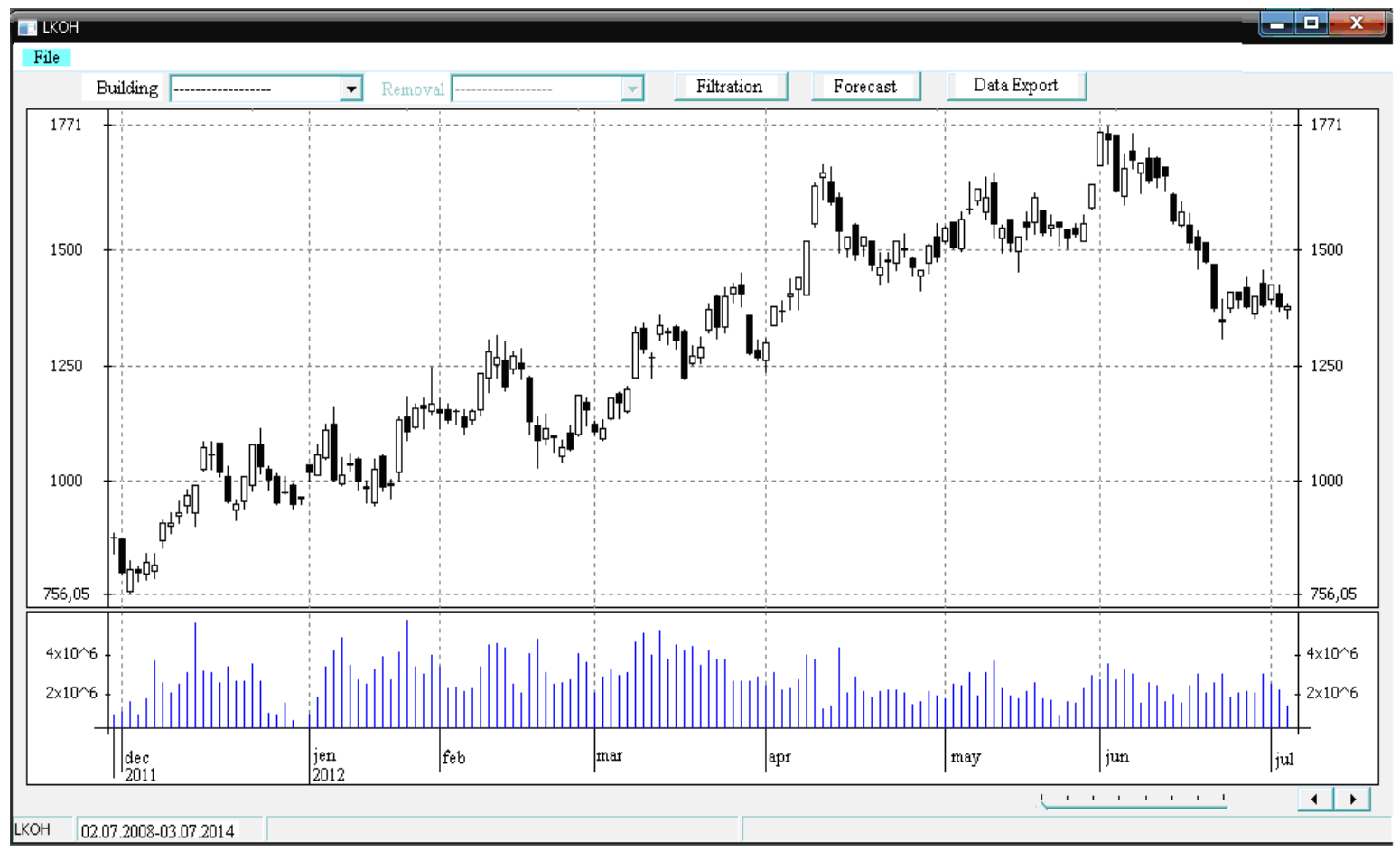

Fig. 3. Example of graphical representation of stock prices.

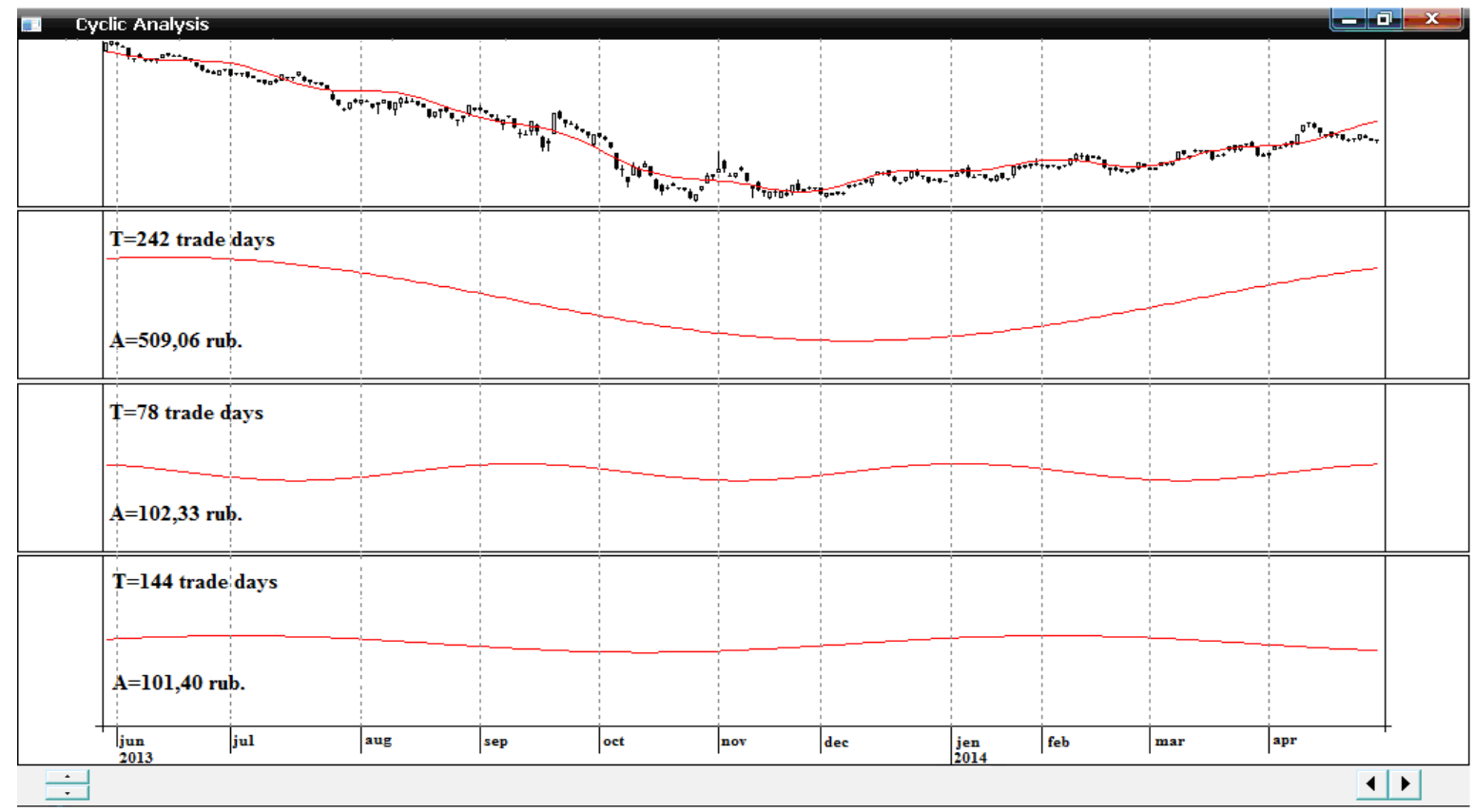

Fig. 4. Example of cyclic analysis with isolation of 3 cycles.

This are a long-term trend of 242 trading days and amplitude of 509 rubles and two mediumterm trends of 78 trading days with amplitude of 102 rubles and 144 trading days with amplitude of 101 rubles.

Analysis of cyclic waves shows that the long-term trend reached its minimum and it is a turning point. At the same time, the medium-term trends reached their highs and are at the same time reducing simultaneously, which should lead to a significant reduction of stock quotes in the short term. 
Thus, it is recommended for long-term investors to buy shares of this issuer after some of their decline, and medium-term investors can be recommended to exit these securities and then enter them after their fall.

Another distinctive feature of the software is building of a forecast indicator based on cyclic analysis. Graphical representation of the developed trade indicator for the dynamics of stock prices of "LUKOIL" for the July-October 2014 is shown in Fig. 5. In accordance with the above-described algorithm the forecast of price values on November (red line) and signals for buying (red arrow) and selling (blue arrows) were obtained.

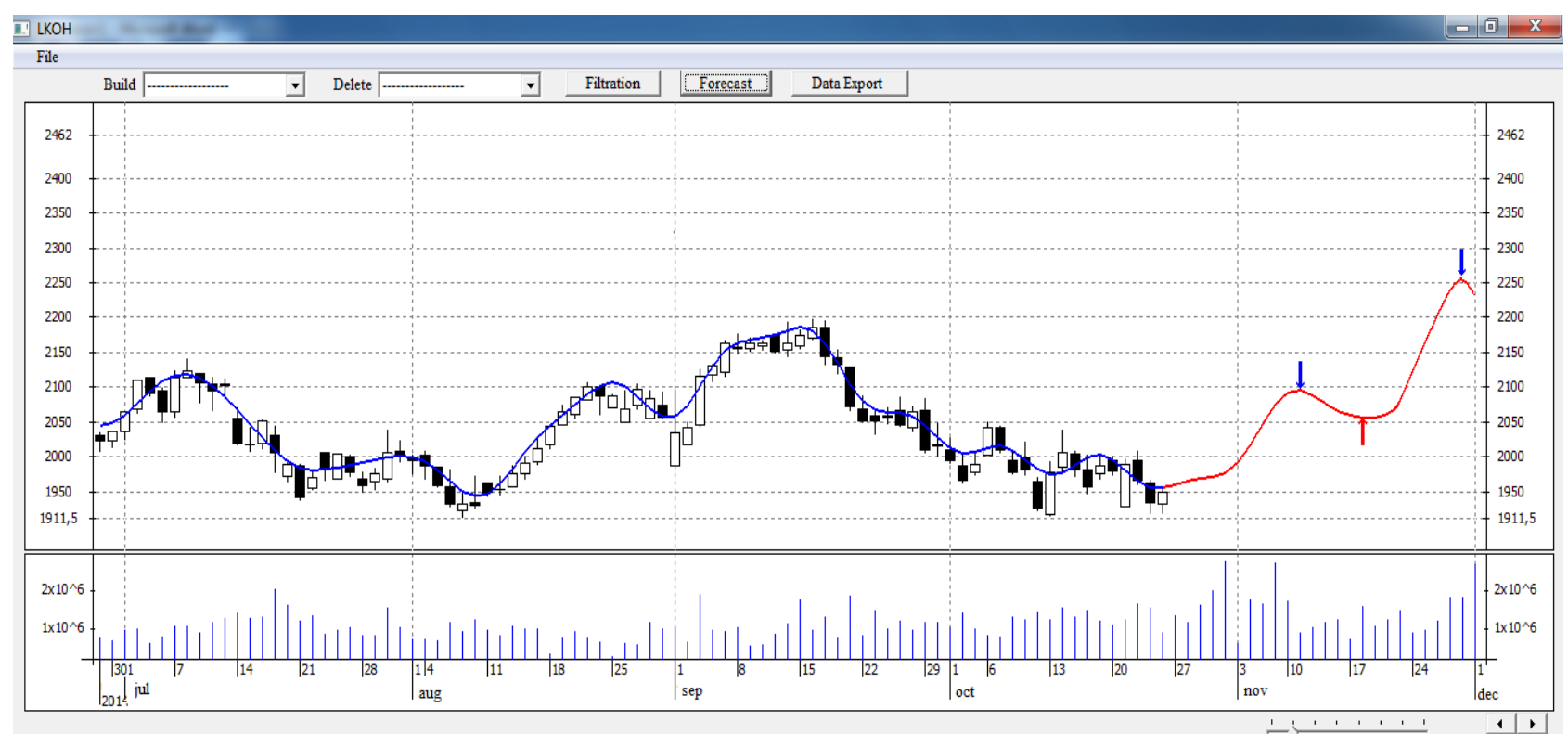

LKOH $01.07 .2013-01.12 .2014 \quad$ Forecasting indicator $(0,01,150)$

Fig. 5. Example of the forecast indicator for LUKOIL stocks.

To check the quality of the forecast the comparing of forecast values with the prices of the shares of OAO "LUKOIL" formed during the trading sessions of the Moscow stock exchange in November 2014 was made (Fig. 6). To obtain the numerical characteristics of the forecast the software calculates the average relative approximation error. For this example, it was $2.704 \%$.

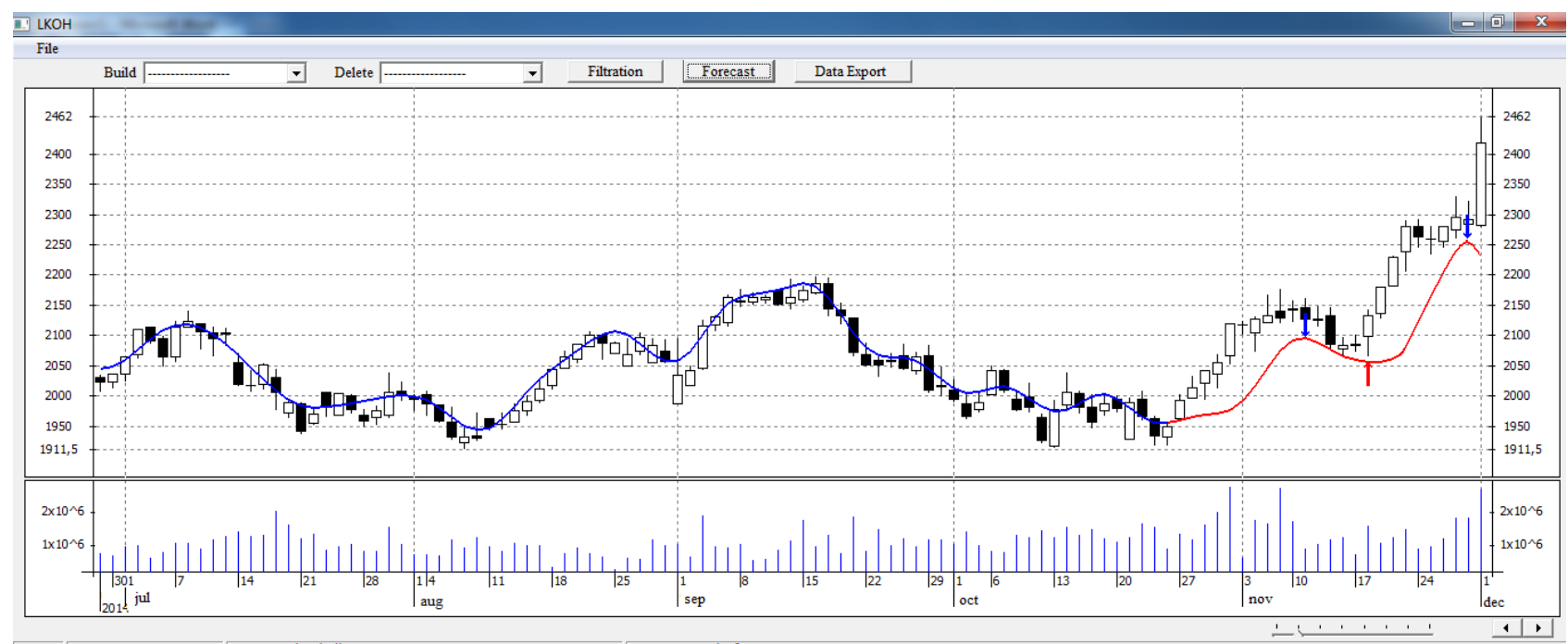

\begin{tabular}{|l|l|l|l|}
\hline $\mathrm{LKOH}$ & $01.07 .2013-01.12 .2014$ & Forecasting indicator $(0,01,150)$ & Average ratio forecast error $2,704 \%$
\end{tabular}

Fig. 6. Example of comparing of forecast with real prices of LUKOIL stocks.

The application of the forecast indicator allows broker or trader to plan upcoming income and expenditure of own funds for their effective using and management of a portfolio of financial assets. 


\section{Conclusions}

The article provides a description of the developed software that allows to use not only standard technical analysis trading indicators, but also a number of additional options that are not implemented by the most common systems "MetaStock", "Omega". Namely, it allows to optimize the parameters of standard technical analysis trading indicators, to determine the signals to buy and sell securities, to calculate expected return on the use of a trading indicator, to analyze quotations of securities with method of cyclic analisys. In addition to the well-known trading indicators software offers a new indicator that allows short-term forecast the dynamics of quotations of securities and on its basis receive the forecast signals to buy or sell.

\section{References}

[1] Sismondi, J. A Neglected Pioneer, History of Political Economy, 4(1), pp.62-88, 1972.

[2] Marx, K. Collected Works of Karl Marx and Frederick Engels, Vol. 35, Vol. 36 and Vol. 37, International Publishers: New York, 1997.

[3] Kitchin, J. Cycles and Trends in Economic Factors. Review of Economics and Statistics (The MIT Press),5 (1), pp. 10-16, 1923.

[4] Schumpeter, J. A. History of Economic Analysis, London: George Allen \& Unwin, 1954.

[5] Frost, A.J.; Prechter, Robert R., Jr., Elliott Wave Principle, 10th ed., Gainesville, GA: New Classics Library, p. 9, 2005.

[6] Murphy, J. Technical Analysis of the Financial Markets, Prentice Hall Press, 1999.

[7] Gujarati, Damodar N.; Porter, Dawn C., Basic Econometrics, 5th ed., Boston: McGraw-Hill Irwin, 2009. 